\title{
A suspected sugammadex-induced anaphylactic shock \\ - A case report -
}

Received October 4, 2018

Revised October 24, 2018

Accepted October 24, 2018

\section{Corresponding author}

Sang Hyun Kim, M.D., Ph.D. Department of Anesthesiology and Pain Medicine, Soonchunhyang University Bucheon Hospital, Soonchunhyang University College of Medicine, 170 Jomaru-ro, Wonmigu, Bucheon 14584, Korea

Tel: 82-32-621-5328

Fax: 82-32-621-5322

E-mail: skim@shcmc.ac.kr

\section{ORCID}

https://orcid.org/0000-0001-6267-7365

\section{Bon Sung Koo, So Jeong Lee, Hyun Woo Na, Woo Bin Kang, and Sang Hyun Kim}

Department of Anesthesiology and Pain Medicine, Soonchunhyang University Bucheon Hospital, Soonchunhyang University College of Medicine, Bucheon, Korea

Background: The reversal of a neuromuscular blockade has typically been achieved with a cholinesterase inhibitor and the concomitant use of an anticholinergic agent, and this remains a popular method. Since the introduction of sugammadex in the market, its use has been increasing because of the rapid recovery from a neuromuscular blockade achieved by rocuronium. The occurrence of anaphylaxis or an anaphylactic reaction resulting from sugammadex is rare and has been reported sparsely. Thus, one may not recognize the possibility of sugammadex-induced hypersensitivity when sudden lifethreatening hypotension occurs, especially without skin manifestations during the emergence of anesthesia. This may delay treatment and increase morbidity.

Case: We report a case of a sugammadex-related hypersensitivity reaction which manifested as pure cardiovascular collapse during the emergence of anesthesia.

Conclusions: We emphasize that vigilance should be paid for at least five minutes following sugammadex administration in daily clinical practice.

Keywords: Anaphylaxis; Hypersensitivity; Sugammadex.

Sugammadex is a $\gamma$-cyclodextrin [1] that is used to reverse aminosteroid neuromuscular blockades, similarly to rocuronium and vecuronium. It was approved for use in the European Union in July 2008, but the United States Food and Drug Administration (FDA) did not approve this drug then because of concerns regarding hypersensitivity. In December 2015, the FDA approved sugammadex in adults undergoing surgery, but they warned that anaphylaxis can occur in $0.3 \%$ of healthy volunteers and recommended that clinicians should be prepared for the possibility of drug hypersensitivity reactions (including anaphylactic reactions) and take necessary precautions. Previous sugammadex hypersensitivity cases reported in Korea have typically presented with both cardiovascular instability and skin lesions. However, there has been no report of sugammadex hypersensitivity that manifested as pure cardiovascular instability without any skin lesions. Pure cardiovascular instability without skin and cutaneous manifestations may delay the diagnosis and treatment when suspected sugammadex-induced anaphylaxis occurs.

Here, we describe the case of a healthy man who developed a severe anaphylactic reaction purely manifested as cardiovascular collapse following the administration of sugammadex during the attempted reversal of rocuronium-induced neuromuscular blockade.

This is an Open Access article distributed under the terms of the Creative Commons Attribution Non-Commercial License (http://creativecommons.org/licenses/by-nc/4.0) which permits unrestricted non-commercial use, distribution, and reproduction in any medium, provided the original work is properly cited. 


\section{CASE REPORT}

A 60-year-old man (body weight $61.4 \mathrm{~kg}$, height $160 \mathrm{~cm}$ ) visited the emergency room for a foreign body in his left eye. He had been nailing with an iron rod when a piece of the iron broke off and entered his eyeball. He was scheduled for emergency surgery with the diagnosis of left-eyeball rupture with intraocular foreign body, retinal detachment, peripheral retinal break, full thickness corneal laceration, and traumatic cataract.

He had no history of allergies and had not undergone any surgery under general anesthesia. The results of all preanesthetic evaluations, including vital signs, laboratory tests, chest radiography, and electrocardiography, were in the normal range, and an emergency operation was planned under general anesthesia.

Routine anesthesia monitoring was conducted, including electrocardiography, pulse oximetry, noninvasive blood pressure, and neuromuscular monitoring. An entropy sensor was attached to monitor the depth of anesthesia. No premedication drug was administered. General anesthesia was induced with $150 \mathrm{mg}$ of propofol and tracheal intubation was facilitated with $50 \mathrm{mg}$ of intravenous rocuronium. In addition, we administered $10 \mathrm{mg}$ of dexamethasone intravenously. Anesthesia was maintained with $6 \%$ desflurane with a total air flow of $2 \mathrm{~L} / \mathrm{min}$ and remifentanil (target controlled infusion $0.1-2 \mathrm{ng} / \mathrm{ml}$ ). Rocuronium was continuously infused so as to prevent possible movement during the operation. Vital signs were stable, and end-tidal $\mathrm{CO}_{2}$ was maintained within normal ranges. The operation time was $102 \mathrm{~min}$. Neuromuscular blockade was reversed by sugammadex $\left(\right.$ Bridion $^{\circledR}$, Merck and Co., USA) $200 \mathrm{mg}$ at the end of surgery. After $2 \mathrm{~min}$ of sugammadex administration, the train of four count was 4 , and the ratio was restored to $100 \%$. However, blood pressure was unmeasurable at this time. We repeated two or three consecutive blood pressure measurement and finally severe hypotension (44/27 mmHg) was found with tachycardia (120 beats/ min). Despite the $100 \%$ oxygen supply, the $\mathrm{SpO}_{2}$ level was 93\%. We administered $100 \mu \mathrm{g}$ of phenylephrine twice along with $5 \mathrm{mg}$ ephedrine with rapid intravenous fluid infusion, but the blood pressure did not increase. We immediately used transthoracic echocardiography to evaluate the unexplained severe hypotension. Cardiac contractility, chamber volume, and valvular function were apparently normal, except for rather hyperdynamic left ventricular contraction. There were no signs of myocardial ischemia, hypovolemia, left ventricular outflow tract obstruction, pulmonary thromboembolism, or pericardial effusion. Thus, we suspected the vasodilatory shock to be related to sugammadex hypersensitivity and administered intravenous bolus of $100 \mu \mathrm{g}$ epinephrine twice along with chlorpheniramine $4 \mathrm{mg}$. Blood pressure then increased from $78 / 48 \mathrm{mmHg}$ to $112 / 54 \mathrm{mmHg}$ in the 20 min following the initial episode of hypotension. However, the blood pressure was not maintained within this range despite bolus administration of epinephrine. We decided to administer norepinephrine so as to maintain blood pressure. We started continuous norepinephrine infusion of $0.1 \mu \mathrm{g} /$ $\mathrm{kg} / \mathrm{min}$; systolic blood pressure was maintained above 110 mmHg with a normalized heart rate. The patient breathed spontaneously and could obey simple commands. We decided to extubate, and the patient was transferred to intensive care for overnight observation. Norepinephrine was tapered within $5 \mathrm{~h}$. The following day, he was transferred to a general ward with normal vital signs and without complications such as cognitive disorder or hypoxic damage. Eight days after the operation, he was discharged.

Table 1. Results of Allergy Skin Tests

\begin{tabular}{llcccc}
\hline \multicolumn{1}{c}{ Type of skin test } & \multicolumn{1}{c}{ Drug } & Concentration & Wheal $(\mathrm{mm})$ & Flare $(\mathrm{mm})$ & Result \\
\hline Skin prick test & Sugammadex & $1: 1$ & $10.9 \times 7.6$ & $57.6 \times 30.9$ & Positive \\
& Rocuronium & $1: 1$ & $2.2 \times 2.1$ & $11.0 \times 12.1$ & Negative \\
Intradermal skin test & Histamine (positive control) & & $11.9 \times 12.0$ & $26.5 \times 30.9$ & $0 \times 0$ \\
& Saline (negative control) & & $0 \times 0$ & $27.6 \times 25.4$ & Positive \\
& Sugammadex & $1: 10,000$ & $11.0 \times 6.5$ & $16.5 \times 30.0$ & Positive \\
& & $1: 1,000$ & $14.3 \times 13.2$ & $0 \times 0$ & Positive \\
& & $1: 100$ & $1: 1.6 \times 12.1$ & $0 \times 0$ & Negative \\
& Rocuronium & $1: 100$ & $0 \times 0$ & Negative \\
\hline
\end{tabular}


About eight weeks after the acute anaphylactic reaction, we conducted allergy skin tests after obtaining informed consent. First, a skin prick test was performed with $1: 1$ solutions of sugammadex (100 mg/ml) and rocuronium (10 mg/ $\mathrm{ml}$ ). A drop of each drug solution was placed on the forearm and immediately pricked. The largest diameter of the wheal was measured $15 \mathrm{~min}$ later (Table 1). The skin prick test for sugammadex showed a positive result as the diameter of the wheal was over $3 \mathrm{~mm}$ (Fig. 1). An intradermal skin test was then conducted with dilutions of sugammadex $(1: 100,1$ : $1,000$, and $1: 10,000)$ and rocuronium $(1: 100$ and $1: 1,000)$. Histamine $(1 \mathrm{mg} / \mathrm{ml})$ and saline were used for positive and negative controls, respectively. A small amount of each drug dilution was injected under the skin, producing a $3 \mathrm{~mm}$ intradermal bleb. After $20 \mathrm{~min}$, wheal and flare reactions were measured in the same manner (Table 1). A positive test was considered to be a response with a wheal diameter of at least $8 \mathrm{~mm}$. The results were positive in all concentrations of sugammadex, but negative in all concentrations of rocuronium (Fig. 1). Based on these skin test results, the patient was strongly suspected with sugammadex-induced anaphylactic shock.

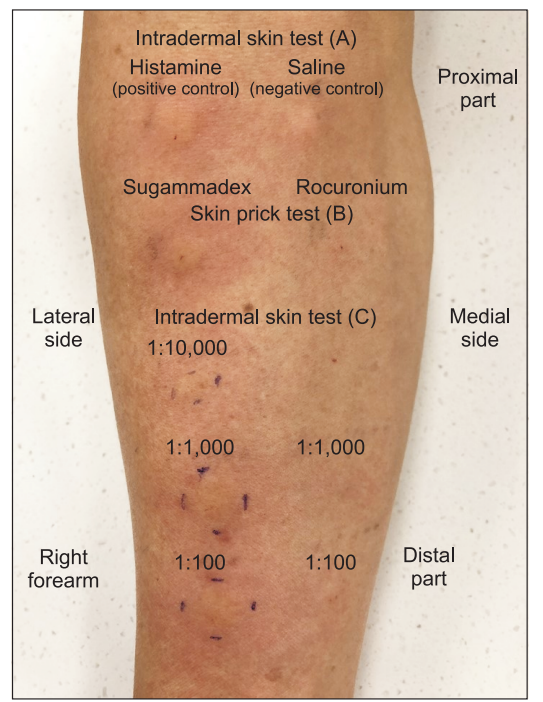

Fig. 1. Skin tests for sugammadex and rocuronium. Histamine (1 mg/ $\mathrm{ml}$ ) and saline were used as positive and negative controls, respectively (A). Skin prick test for sugammadex (1: 1 solution) showed a positive result as the diameter of the wheal was over $3 \mathrm{~mm}$, but a negative result for rocuronium (B). Intradermal skin test with dilutions of sugammadex $(1: 100,1: 1,000$, and $1: 10,000)$ showed positive results as the diameter of the wheal was over $8 \mathrm{~mm}(\mathrm{C})$.

\section{DISCUSSION}

The reversal of rocuronium-induced neuromuscular blockade by administering sugammadex has become popular after the introduction of this agent in the market because of the rapid recovery and fewer side effects. However, sugammadex is not without risk in terms of drug-induced hypersensitivity, and it can be life-threatening.

Anaphylaxis is defined as a life-threatening hypersensitivity reaction with systemic allergic symptoms. Neuromuscular blocking drugs, latex, and antibiotics are the most common causes of perioperative anaphylaxis [2]. Since the World Allergy Organization (WAO) suggested the clinical criteria for diagnosing anaphylaxis [3] in 2011, many studies have taken this guideline for the perioperative diagnosis of anaphylaxis.

Some case reports of anaphylactic reactions with sugammadex have been sporadically reported in many countries [1,4-6]; however, there have been few systematic reviews about sugammadex-related anaphylaxis. Recently, Miyazaki et al. [7] retrospectively investigated the incidence of anaphylaxis potentially caused by sugammadex and reported that the incidence was $0.039 \%$ (six cases among 15,479 patients who received sugammadex; 95\% confidence interval 0.014 $0.084 \%$ ). The most frequently shared signs and symptoms were rash, hypotension, and tachycardia, in decreasing order of incidence [4].

To the best of our knowledge, this is the third case of anaphylaxis associated with sugammadex hypersensitivity in Korea. Our case has features similar to those of the two cases previously reported, except for the fact that our patient had no skin lesion.

Miyazaki et al. [7] reported six cases of sugammadex anaphylaxis, two of which also had no skin lesion, similar to our case. Skin or mucosal lesion is not necessary for the diagnosis of anaphylaxis according to the WAO guideline for the assessment and management of anaphylaxis [3].

Just 2 min after the administration of sugammadex, patient showed hypotension, tachycardia, and hypoxemia that did not involve skin or mucosal tissue. However, the other signs and symptoms were sufficient to suspect and diagnose anaphylaxis despite the absence of rash or erythema. If a patient's symptoms suggest anaphylaxis and occurred after the drug administration, he or she should be treated as appropriate for anaphylaxis. 
In the current case, we immediately used rescue transthoracic echocardiography, and could exclude other causes of acute hypotension such as ischemic heart disease and cardiogenic shock. The immediate use of transthoracic echocardiography is very helpful, especially in clinical settings. The exclusion of other possible causes of unexplained hypotension that require immediate specific treatment allowed us to treat sugammadex-induced anaphylactic shock with confidence.

In their six cases, Miyazaki et al. [7] reported the recovery time as ranging from 4 to $30 \mathrm{~min}$. In our case, it took about $40 \mathrm{~min}$ to achieve hemodynamic stability from the initial hypotensive episode with bolus administration of epinephrine and additional continuous infusion of norepinephrine.

Many case reports about sugammadex hypersensitivity showed fast recovery, and most of these patients were discharged to the postanesthetic care unit and a general ward. However, in this case, the patient required norepinephrine infusion to maintain blood pressure in the normal range, so he was transferred to the intensive care unit for close observation.

The etiological diagnosis of perioperative anaphylaxis relies on a triad of evidence, including clinical, biological, and allergological evidence [8].

The first line of evidence for diagnosing anaphylaxis includes clinical signs [1]. Our case fulfilled the WAO clinical criteria [3], as evidenced by the acute onset of symptoms with hypoxemia and hypotension.

Biological assessment, including histamine and tryptase measurement, is the second line of evidence for diagnosing anaphylaxis [9]. The histamine concentration in blood can be measured a few min after degranulation. Tryptase only originates from mast cells, and increased $\beta$-tryptase concentrations are correlated with decreased arterial blood pressure and associated with an allergic mechanism [9]. However, serum and urine histamine tests are not available in Korea [5].

Skin tests, the third line of evidence, remain the gold standard for the detection of reactions mediated by immunoglobulin E [1]. Intradermal skin test or skin prick tests are usually performed four to six weeks after a reaction, because the intracellular stocks of histamine and other mediators are still lower than normal before the initial four-week period [2]. In our case, the same reasoning was applied. In previous reports, either a skin prick test $[5,10,11]$ or an intradermal skin test [12] was performed in order to detect hypersensitivity to sugammadex. However, there are no established guidelines about skin testing for hypersensitivity to sugammadex $[6,12]$. In cases of skin tests for perioperative drugs, investigations should include a skin prick and/or an intradermal test with all substances to which the patient was exposed [13]. In our case, both the skin prick test and intradermal test were performed with sugammadex and rocuronium, which are among the drugs most likely to be exposed to patients. Mertes et al. [2] reported the optimal concentration of both skin prick test and intradermal test for neuromuscular blocking agents. Based on this report [2], we used a $1: 1$ solution of rocuronium and dilutions of $1: 100$ and $1: 1,000$ of rocuronium for the skin prick test and intradermal test, respectively. However, to the best of our knowledge, no optimal concentration guideline of skin tests for sugammadex has been reported. Therefore, based on a previous report $(1: 100)$ [12], we chose relatively low concentrations $(1: 1,000,1: 10,000)$ of sugammadex for the intradermal test in order to avoid false-positive reactions. We used histamine and saline as positive and negative controls, respectively. The histamine control should be positive so as to ensure that the test materials are applied correctly [14]. The negative control excludes the presence of dermographism, which, when present, makes the tests difficult to interpret [14].

When skin tests results are reported, a wide array of interpretations may be described. Rueff et al. [15] reported that skin tests should be regarded positive if the mean wheal diameter is $\geq 3 \mathrm{~mm}$ in the prick test and $\geq 5 \mathrm{~mm}$ in the intradermal test. By contrast, Mertes et al. [2] reported that a prick test should be considered positive when the diameter of the wheal is at least equal to half of that produced by the positive control test and at least $3 \mathrm{~mm}$ greater than the negative control, and intradermal tests should be considered positive when the diameter of the wheal is twice or more the diameter of the injection wheal. However, there is no guideline of interpretations to be widely used to skin tests for sugammadex. In our case, we did not use controls with the skin prick test. Therefore, we decided that the wheal diameter of more than $3 \mathrm{~mm}$ is a positive finding. In interpreting the intradermal skin test result, based on a previous study [12], a positive test was considered to be a response with a wheal diameter of at least $8 \mathrm{~mm}$.

Although the serum tryptase and histamine measurement 
was not performed, we could suspect sugammadex-induced anaphylaxis based on the positive results of the intradermal skin test for sugammadex.

In summary, we report a case of sugammadex-induced anaphylactic shock which manifested as pure cardiovascular collapse during the emergence of anesthesia. This was strongly supported by a positive intradermal skin test to sugammadex. We recommend that vigilance should be paid for at least five minutes following sugammadex administration. Furthermore, the immediate use of rescue transthoracic echocardiography may helpful in the treatment process.

\section{CONFLICTS OF INTEREST}

No potential conflict of interest relevant to this article was reported.

\section{ORCID}

Bon Sung Koo: https://orcid.org/0000-0003-1578-6950

So Jeong Lee: https://orcid.org/0000-0002-8935-2998

Hyun Woo Na: https://orcid.org/0000-0001-9774-0686

Woo Bin Kang: https://orcid.org/0000-0002-7708-8579

\section{REFERENCES}

1. Takazawa T, Mitsuhata H, Mertes PM. Sugammadex and rocuronium-induced anaphylaxis. J Anesth 2016; 30: 290-7.

2. Mertes PM, Lambert M, Guéant-Rodriguez RM, Aimone-Gastin I, Mouton-Faivre C, Moneret-Vautrin DA, et al. Perioperative anaphylaxis. Immunol Allergy Clin North Am 2009; 29: 429-51.

3. Simons FE, Ardusso LR, Bilò MB, El-Gamal YM, Ledford DK, Ring J, et al. World allergy organization guidelines for the assessment and management of anaphylaxis. World Allergy Organ J 2011; 4: 13-37.

4. Tsur A, Kalansky A. Hypersensitivity associated with sugamma- dex administration: a systematic review. Anaesthesia 2014; 69: 1251-7.

5. Yoo JH, Kim SI, Ok SY, Park SY, Cho A, Han YM, et al. Suspected anaphylactic reaction associated with sugammadex: a case report. Korean J Anesthesiol 2016; 69: 413-6.

6. Hwang MH, Won YJ, Lee IO, Koo EH, Jung WJ. A suspected case of sugammadex-induced anaphylactic shock: a case report. Anesth Pain Med 2015; 10: 288-90.

7. Miyazaki Y, Sunaga H, Kida K, Hobo S, Inoue N, Muto M, et al. Incidence of anaphylaxis associated with sugammadex. Anesth Analg 2018; 126: 1505-8.

8. Dewachter P, Mouton-Faivre C, Emala CW. Anaphylaxis and anesthesia: controversies and new insights. Anesthesiology 2009; 111: 1141-50

9. Laroche D, Gomis P, Gallimidi E, Malinovsky JM, Mertes PM. Diagnostic value of histamine and tryptase concentrations in severe anaphylaxis with shock or cardiac arrest during anesthesia. Anesthesiology 2014; 121: 272-9.

10. Menéndez-Ozcoidi L, Ortiz-Gómez JR, Olaguibel-Ribero JM, Salvador-Bravo MJ. Allergy to low dose sugammadex. Anaesthesia 2011; 66: 217-9.

11. Godai K, Hasegawa-Moriyama M, Kuniyoshi T, Kakoi T, Ikoma K, Isowaki $\mathrm{S}$, et al. Three cases of suspected sugammadex-induced hypersensitivity reactions. Br J Anaesth 2012; 109: 216-8.

12. Sadleir PH, Russell T, Clarke RC, Maycock E, Platt PR. Intraoperative anaphylaxis to sugammadex and a protocol for intradermal skin testing. Anaesth Intensive Care 2014; 42: 93-6.

13. Brockow K, Garvey LH, Aberer W, Atanaskovic-Markovic M, Barbaud A, Bilo MB, et al. Skin test concentrations for systemically administered drugs -- an ENDA/EAACI Drug Allergy Interest Group position paper. Allergy 2013; 68: 702-12.

14. Heinzerling L, Mari A, Bergmann KC, Bresciani M, Burbach G, Darsow U, et al. The skin prick test - European standards. Clin Transl Allergy 2013; 3: 3.

15. Ruëff F, Bergmann KC, Brockow K, Fuchs T, Grübl A, Jung K, et al. Skin tests for diagnostics of allergic immediate-type reactions. Guideline of the German Society for Allergology and Clinical Immunology. Pneumologie 2011; 65: 484-95. 